\title{
Design of Two-Channel Linear Phase Orthogonal Cyclic Filterbanks
}

\author{
Ravi Motwani and K. R. Ramakrishnan
}

\begin{abstract}
In this letter, we propose a method to design an orthonormal two-channel cyclic filterbank (CFB) with real analysis and synthesis filters having linear phase. This is particularly significant, since it is not possible to realize regular two-channel linear phase orthogonal filterbanks (unless the filters are two-tap). The lowpass filter of the FB is obtained as a spectral factor of a zero phase Nyquist(2) filter, which is optimized. We show that the CFB design is computationally less demanding as compared to the noncyclic case, and it may be possible to get better filters. We design the CFB for a particular case and show that this filterbank is not a noncyclic orthogonal filterbank.
\end{abstract}

Index Terms - Cyclic filterbanks, paraunitary filterbanks, transform coders.

\section{INTRODUCTION}

I $\mathrm{N}$ THIS letter we consider the design of two-channel orthogonal cyclic filterbank (CFB) shown in Fig. 1. The central problem in the design of this perfect reconstruction (PR) system is to design the analysis filters under the constraint that $\mathbf{E}(k)$ (Fig. 2) is paraunitary. With $\mathbf{E}(k)$ constrained to be paraunitary we try to minimize the sum of stopband energies

$$
\sum_{k=0}^{1} \sum_{l \in P_{k}}\left|H_{k}(l)\right|^{2}
$$

where $P_{k}$ is the stopband of $H_{k}$, by optimizing the parameters characterizing $\mathbf{E}(k)$. The passbands of $H_{k}(l)$ automatically turn out to be good as will be shown.

We assume throughout the letter that the input signal is periodic with period $L$, which is even, i.e., $L=2 K$ (pad a zero if not).

\section{Properties of Orthogonal CFB}

The CFB (Fig. 1) is said to be orthogonal if

$$
\sum_{n=0}^{L-1} h_{i}(n) h_{j}(n-2 l)=\delta(i-j) \delta(l)
$$

(all arguments are to be interpreted modulo the periodicity of the signal).

Manuscript received December 10, 1997. The associate editor coordinating the review of this manuscript and approving it for publication was Prof. A. Tewfik.

The authors are with the Department of Electrical Engineering, Indian Institute of Science, Bangalore, India 560012 (e-mail: motwa@ee.iisc.ernet.in; krr@ee.iisc.ernet.in).

Publisher Item Identifier S 1070-9908(98)03725-0.

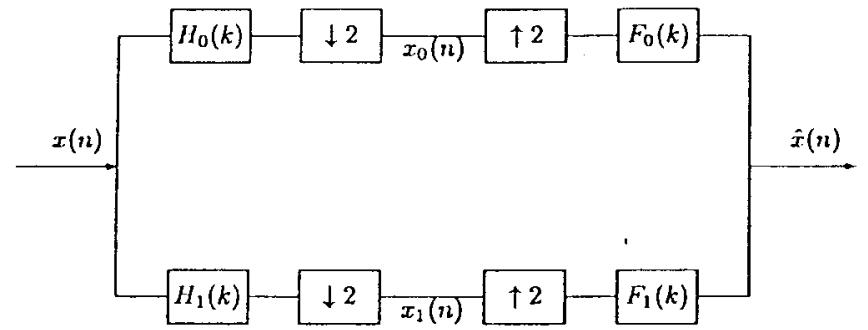

Fig. 1. Cyclic filterbank.

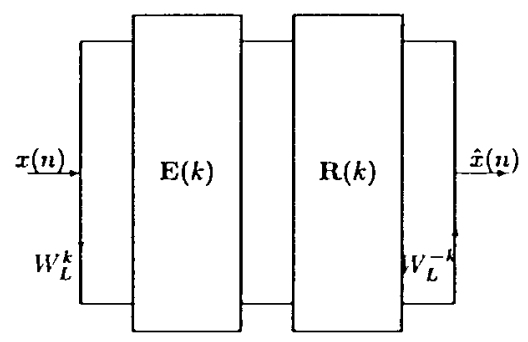

Fig. 2. Equivalent representation.

Orthogonality of the CFB's is equivalent to $\mathbf{E}(k)$ being paraunitary [2]. The paraunitariness of $\mathbf{E}(k)$ is equivalent to

- $H_{0}(l)$ and $H_{1}(l)$ are spectral factors of Nyquist(2) filters [1];

- $\left|H_{0}(l)\right|^{2}+\left|H_{1}(l)\right|^{2}=1,0 \leq l \leq L-1$

(this shows that if the stopband energies are minimized the passbands also will turn out to be good);

- $H_{0}^{*}(l) H_{0}(l+K)+H_{1}^{*}(l) H_{1}(l+K)=0,0 \leq l \leq K-1$;

- $H_{0}^{*}(l) H_{1}(l)+H_{0}^{*}(l+K) H_{1}(l+K)=0, \quad 0 \leq l \leq K-1$.

For an orthogonal CFB, PR is possible iff $\mathbf{R}(k)=\mathbf{E}^{\dagger}(k), 0 \leq$ $k \leq K-1$, i.e., the synthesis filters are chosen as $F_{0}(k)=$ $H_{0}^{*}(k), F_{1}(k)=H_{1}^{*}(k), \quad 0 \leq k<L$.

If $H_{1}(l)=-W_{L}^{k} H_{0}^{*}(l-K)$, and $H_{0}(l)$ is selected as a spectral factor of a Nyquist(2) filter, $\mathbf{E}(k)$ will turn out to be paraunitary from the above equivalence.

\section{FILTER DESIGN}

From the above section, we know that if $H_{1}(k)$ is selected as above, then only $H_{0}(k)$ remains to be designed, since all the other filters are determined. The ideal filters would be

$$
\begin{aligned}
& H_{0}(k)= \begin{cases}1, & -\frac{L}{4}<k<\frac{L}{4}+1, \\
0, & \text { otherwise }\end{cases} \\
& H_{1}(k)= \begin{cases}0, & -\frac{L}{4}<k<\frac{L}{4}+1 \\
1, & \text { otherwise. }\end{cases}
\end{aligned}
$$



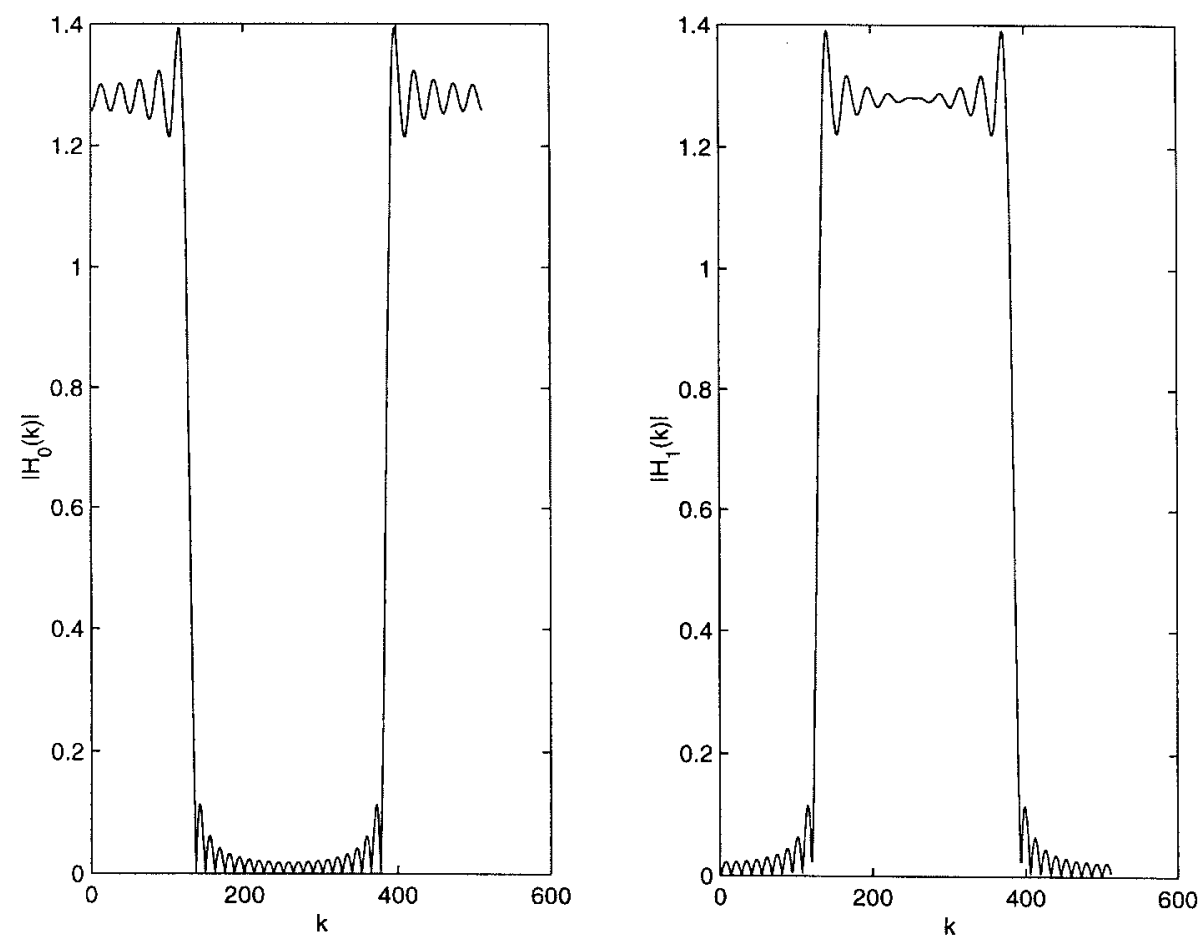

Fig. 3. Magnitude response of lowpass and highpass filters for $N=20$.

But these filters are complex. To obtain real filters, we pose the filter design problem as an optimization problem, by imposing the constraint that the filter be real and try to minimize the stopband energy. We know from Section II that minimizing the stopband energy ensures a good passband. Since we obtain $H_{0}(k)$ as a spectral factor of a real Nyquist(2) filter, we design a zero phase Nyquist(2) filter and optimize it to minimize its stopband energy.

Let

$$
G(k)=\sum_{n=0}^{L-1} g(n) W_{L}^{n k}
$$

denote the Nyquist(2) filter $g(n)=g(-n)$

Let

$$
\begin{aligned}
G(k) & =g(0)+\sum_{n \text { odd, }, 0<n<L} g(n) W_{L}^{n k} \\
& =g(0)+\sum_{n \text { odd, }, 0<n<\frac{L}{2}} 2 g(n) \cos \left(\frac{2 \pi k n}{L}\right) .
\end{aligned}
$$

$$
g=\left[\begin{array}{lllll}
g(0) & g(1) & g(3) & \cdots & g(J)
\end{array}\right]^{t} \quad g(i) \text { real }
$$

where $J$ is the largest odd integer less than $\frac{L}{2}$.

Let

$$
\stackrel{c(k)}{=}\left[12 \cos \left(\frac{2 \pi k}{L}\right) 2 \cos \left(\frac{2 \pi 3 k}{L}\right) \cdots 2 \cos \left(\frac{2 \pi J k}{L}\right)\right] \cdot{ }^{t}
$$

In this case, the set of points $P_{s}=\left\{\frac{L}{4}+1, \frac{L}{4}+2 \cdots 3 \frac{L}{4}\right\}$ constitutes the stopband. The problem now reduces to finding a real vector $\mathbf{g}$ such that the stopband energy of $G(k)$ is minimized.

$$
\begin{aligned}
\min _{\mathbf{g}} \sum_{k \in P_{s}}|G(k)|^{2} & =\min _{\mathbf{g}} \sum_{k \in P_{s}} \mathbf{g}^{t} \mathbf{c}(k) \mathbf{c}^{t}(k) \mathbf{g} \\
& =\min _{\mathbf{g}} \mathbf{g}^{t} \mathbf{R g}
\end{aligned}
$$

where $\mathbf{R}=\sum_{k \in P_{s}} \mathbf{c}(k) \mathbf{c}^{t}(k)$ is a real symmetric, positive definite matrix. Then the solution to above problem is the eigenvector corresponding to the smallest eigenvalue of $\mathbf{R}[1]$.

Define

$$
G^{\prime}(k)=G(k)-\min _{k} G(k) .
$$

Then

$$
H_{0}(k):=\sqrt{G^{\prime}(k)}
$$

is the required lowpass filter.

Alternatively, we can also select $H_{0}(k)=e^{j \phi(k)} \sqrt{G(k)}$, where $\phi(k)$ can be selected, such that $H_{0}(k)$ is real.

It can be shown that $H_{0}(k)=H_{0}(-k)$, and since $H_{0}(k)$ is real, the filter $h_{0}$ is real and symmetric, i.e., $h_{0}(n)=h_{0}(-n)$.

Advantages of Orthogonal CFB: In the design of noncyclic FB, there is an additional computational overhead in arriving at the spectral factor of the Nyquist filter. It involves the computation of the cepstrum and its inverse $z$ transform, whereas for $\mathrm{CFB}$, we have to just perform the square root operation. Furthermore, the computation of the matrix $\mathbf{R}$ in the previous section is easier for the cyclic case than the noncylic case, which involves numerical integration. It can be shown that all the filters are linear phase power symmetric filters, something that is not possible to obtain in the noncyclic case. 

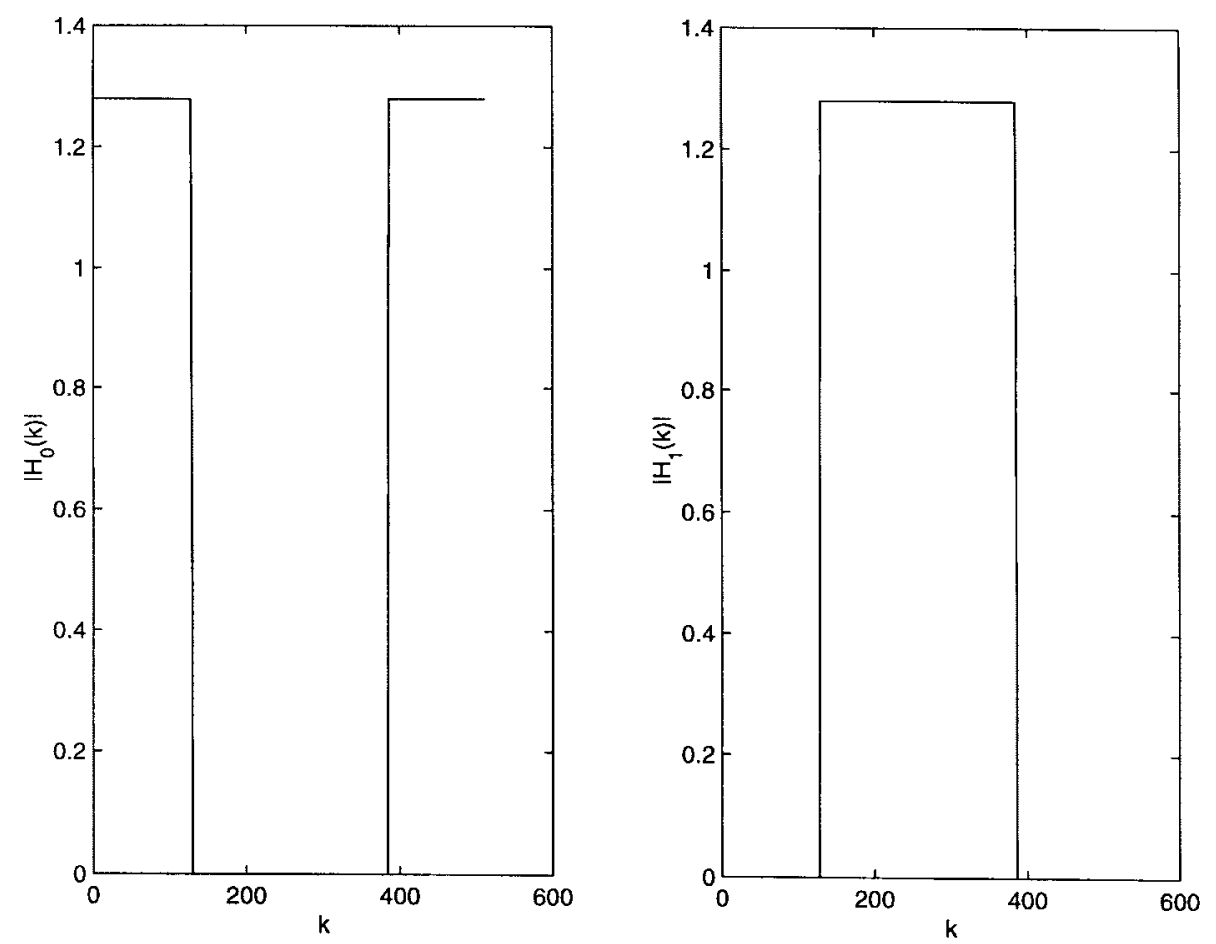

Fig. 4. Magnitude response of lowpass and highpass filters for $N=L$.

\section{A DESIGN EXAMPLE}

We design an orthogonal system with real coefficient analysis and synthesis filters for $L=512$. We first design a zero phase Nyquist(2) filter $G(z)$, with the constraint that the stopband energy is minimized. The lowpass filter is then obtained as $H_{0}(k)=\sqrt{G(k)-\min _{k} G(k)}$. The other filters are obtained from $H_{0}(k)$ as discussed in the Section III. Fig. 3 shows the magnitude responses of the lowpass and the highpass filters, obtained by truncating the actual filters obtained with a window of length $N=20$, the window function satisfying $w(n)=w(-n)$. As we increase the window size, the response becomes better. Fig. 4 shows the responses of the lowpass and the highpass filters with length of the window $N=L . H_{0}$ is a zerophase filter and all the other filters are linear phase. It is easy to show that this orthogonal CFB is not an orthogonal noncyclic FB. We know that in a noncyclic two-channel orthogonal FB the following relation holds [3]:

$$
\left\langle h_{i}(n), h_{j}(n-2 l)\right\rangle=\delta(i-j) \delta(l) \forall l, i, j=0,1 .
$$

It can be shown that this relation does not hold for the above FB.

\section{CONCLUSIONS AND Future SCOPE}

In this letter, we design a two-channel linear phase orthogonal CFB and show that it may give better filters for finite length signals than noncylic orthogonal FB. This is particularly useful for applications like image processing, where it is desirable to use linear phase orthogonal FB's. One can look for obtaining a proper methodology for designing $M$ channel orthogonal CFB for $M>2$.

\section{REFERENCES}

[1] P. P. Vaidyanathan, Multirate Systems and Filter Banks. Englewood Cliffs, NJ: Prentice-Hall, 1993.

[2] P. P. Vaidyanathan and A. Kirac, "Theory of cyclic filter banks," in Proc. ICASSP'97.

[3] M. Vetterli and C. Herley, "Wavelets and filter banks-Theory and design," IEEE Trans. Signal Processing, vol. 40, pp. 2207-2232, Sept. 1992.

[4] P. P. Vaidyanathan, T. Nguyen, Z. Doganata, and T. Saramaki, "Improved technique for design of PR FIR QMF banks with lossless polyphase matrices," IEEE Trans. Acoust., Speech, Signal Processing, vol. 37, pp. 1042-1056, July 1989. 